MALARIA

JOURNAL

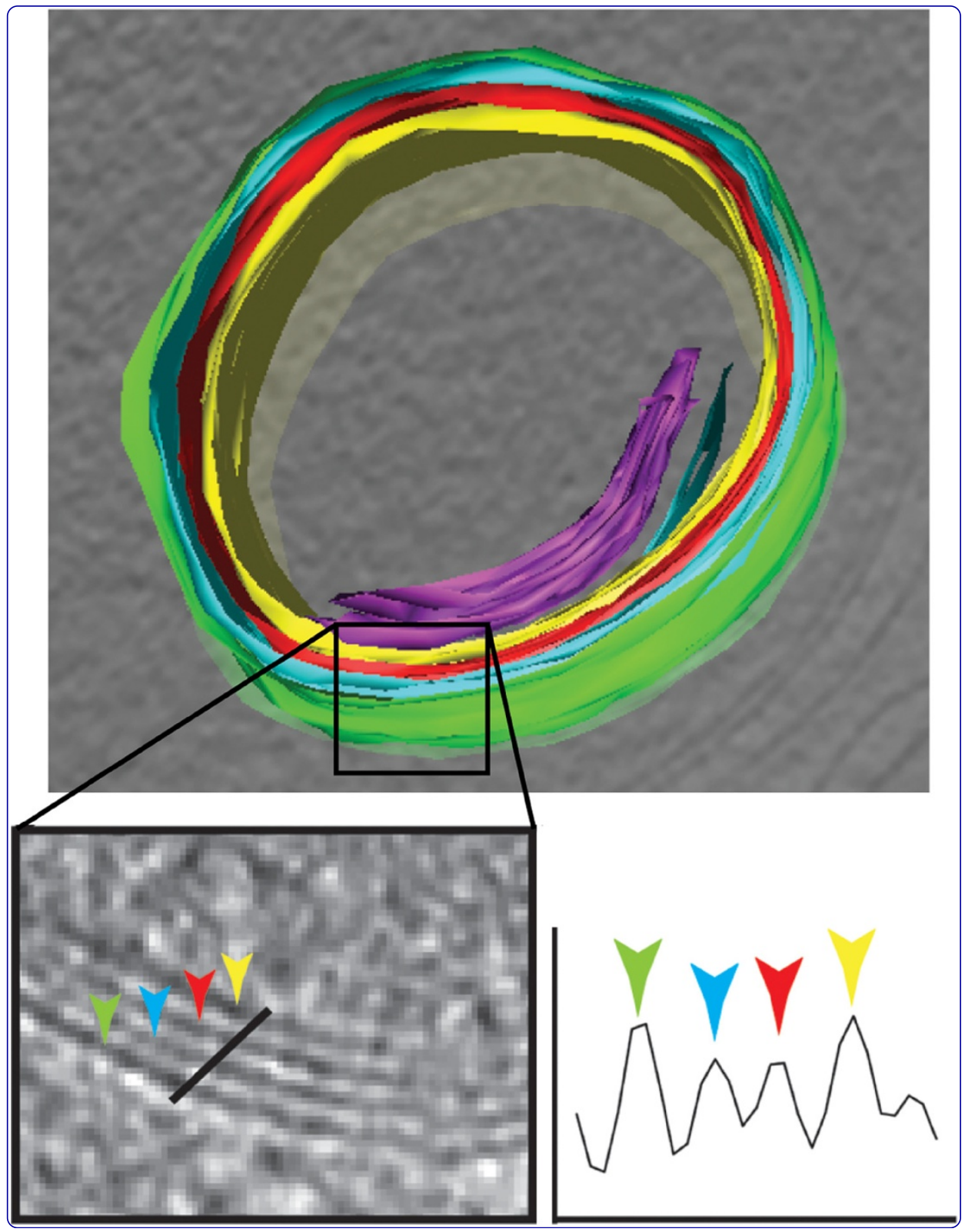

\title{
Cryo-electron tomography reveals four-membrane architecture of the Plasmodium apicoplast
}

Lemgruber et al.

C BioMed Central 


\title{
Cryo-electron tomography reveals four-membrane architecture of the Plasmodium apicoplast
}

\author{
Leandro Lemgruber ${ }^{1 \dagger}$, Mikhail Kudryashev ${ }^{2 \dagger}$, Chaitali Dekiwadia ${ }^{3}$, David T Riglar ${ }^{4,5}$, Jake Baum ${ }^{4,5}$, \\ Henning Stahlberg ${ }^{2}$, Stuart A Ralph ${ }^{3^{*}}$ and Friedrich Frischknecht ${ }^{1 *}$
}

\begin{abstract}
Background: The apicoplast is a plastid organelle derived from a secondary endosymbiosis, containing biosynthetic pathways essential for the survival of apicomplexan parasites. The Toxoplasma apicoplast clearly possesses four membranes but in related Plasmodium spp. the apicoplast has variably been reported to have either three or four membranes.

Methods: Cryo-electron tomography was employed to image merozoites of Plasmodium falciparum and Plasmodium berghei frozen in their near-native state. Three-dimensional reconstructions revealed the number of apicoplast membranes and the association of the apicoplast with other organelles. Routine transmission electron microscopy of parasites preserved by high-pressure freezing followed by freeze substitution techniques was also used to analyse apicoplast morphology.

Results: Cryo-preserved parasites showed clearly four membranes surrounding the apicoplast. A wider gap between the second and third apicoplast membranes was frequently observed. The apicoplast was found in close proximity to the nucleus and to the rhoptries. The apicoplast matrix showed ribosome-sized particles and membranous whorls.

Conclusions: The Plasmodium apicoplast possesses four membranes, as do the apicoplasts of other apicomplexan parasites. This is consistent with a four-membraned secondary endosymbiotic plastid ancestor.
\end{abstract}

Keywords: Apicoplast, Cryo-electron tomography, Symbiosis, Organelle interaction, Malaria

\section{Background}

All modern plastids are remnants of a primary endosymbiosis that occurred around one billion years ago, whereby a cyanobacterium was engulfed and retained by a eukaryote. The algae produced by this event possess plastids with two membranes corresponding to the double membranes of the gram-negative cyanobacteria. These algae later diverged into groups known today as Viridiplantae, Glaucophyta and Rhodophyta, and their plastids are known as primary endosymbionts. All

\footnotetext{
* Correspondence: saralph@unimelb.edu.au; freddy.frischknecht@med.uniheidelberg.de

${ }^{\dagger}$ Equal contributors

${ }^{3}$ Department of Biochemistry and Molecular Biology, Bio21 Molecular Science and Biotechnology Institute, The University of Melbourne, Victoria 3010,

Australia

${ }^{1}$ Parasitology, Department of Infectious Diseases, University of Heidelberg Medical School, Im Neuenheimer Feld 324, Heidelberg 69120, Germany

Full list of author information is available at the end of the article
}

existing plastids are either found in these algae, or in later eukaryotes that phagocytosed plastid-bearing organisms and retained their enclosed plastid [1]. Plastids acquired through capture of an organism that contained a primary endosymbiont are referred to as secondary endosymbionts. These organelles would initially have possessed four membranes, deriving from the two primary endosymbiont membranes, plus the plasma membrane of the primary endosymbiont and the phagosomal membrane of the engulfing eukaryote. In some lineages, one of those outer membranes has been secondarily lost to give rise to three-membrane plastids [2]. Algae with secondary endosymbionts can themselves be captured and retained (tertiary endosymbiosis) giving rise to plastids with more than four membranes [3]. 
Although all extant plastids are believed to derive from a single primary endosymbiosis event, secondary endosymbiosis has occurred a number of times, giving rise to multiple, unrelated plastid-bearing groups. One such hypothesized group is the chromalveolata, proposed to have arisen from a single secondary endosymbiosis involving a rhodophyte (red alga), that gave rise to extremely diverse organisms such as diatoms, dinoflagellates and apicomplexans [4].

The plastid of apicomplexan parasites - the apicoplast - has received considerable attention, in part because of the evolutionary implications of the presence of a plastid in this phylum [5,6], but mainly because apicomplexans are of immense medical and veterinary significance and the apicoplast is the target for important antiparasitic drugs [7]. If the chromalveolate hypothesis holds, the common ancestor of all apicomplexans possessed a fourmembrane plastid, although at least one apicomplexan genus, Cryptosporidium, has subsequently lost its plastid. Apicoplasts lack a photosynthetic apparatus, but a recently identified close relative of the Apicomplexa, the alga Chromera [8], is photosynthetic and possesses a four-membrane plastid that appears to derive from the same red algal origin as the apicoplast [9].

Descriptions of apicoplast membranes are many and varied. Because the apicoplast identity was established long after apicomplexans had been ultrastructurally investigated there are many descriptions of the apicoplast with differing generic names, eg, spherical body, hohlzylinder and Golgi adjunct. Micrographs since the description of the apicoplast establish plastids with four membranes for many of the apicomplexans, including Toxoplasma gondii [10], Sarcocystis [11], Garnia gonadati [12], and Babesia bovis [13] as do many of the older micrographs for diverse apicomplexans (discussed in [14]). However, descriptions of the apicoplast of Plasmodium species, the causative agents of malaria, are conflicting. Some preparations appear to support a four-membrane interpretation in sporozoites [15]; however this was based on a single observation and thus was not further commented upon. In contrast other electron micrographs suggest an apicoplast with only three membranes [16-18]. This has led to an ongoing disagreement in the number of apicoplast membranes of Plasmodium. Loss of one of the four membranes clearly found in other apicomplexans would have considerable molecular consequences for understanding protein trafficking and biogenesis for the Plasmodium apicoplast, so the resolution of this question is desirable.

To investigate this discrepancy, electron microscopy of cryopreserved parasites from Plasmodium falciparum and Plasmodium berghei coupled to tomographic reconstructions was used. Cryo-electron tomography is widely regarded to introduce the fewest artefacts during preparation as the specimen is rapidly frozen (within a few milliseconds) thus preserving molecular details and membrane arrangements $[19,20]$. This technique has been used successfully to investigate membranous and cytoskeletal structures in sporozoites of $P$. berghei [15,21-23] and Maurer's clefts of P. falciparum infected red blood cells $[24,25]$. Cryo-electron tomography does not include staining with heavy metal salts and thus provides lower contrast, though being sufficient to examine membranes [26]. Reconstructed tomograms of both P. falciparum and P. berghei merozoites clearly show the apicoplast with four delimiting membranes. These membranes often appear paired with a gap between the second and third membranes. In some individual sections through the tomograms, only three membranes are apparent around some apicoplasts, but three-dimensional reconstructions of these resolves the local appositions of two membranes to a total of four delimiting membranes.

\section{Methods}

\section{Ethics statement}

All animal experiments were performed according to the FELASA and GV-SOLAS standard guidelines. Animal experiments were approved by the German authorities (Regierungspräsidium Karlsruhe).

\section{Obtaining Plasmodium merozoites}

Plasmodium falciparum parasites from strains 3D7 and D10 were maintained using standard procedures. Cultures were grown in human erythrocytes in RPMI 1640 supplemented with L-glutamine, HEPES, hypoxanthine, and gentamycin. Some cultures were supplemented with human heat-inactivated serum and albumax, and others were supplemented with albumax alone. Parasites were incubated at $37^{\circ} \mathrm{C}$ in a gas mixture of $5 \%$ $\mathrm{CO}_{2}, 1 \% \mathrm{O}_{2}$, and $94 \% \mathrm{~N}_{2}$. For merozoites subject to cryopreservation prior to electron tomography, late schizonts were harvested by magnetic cell sorting and resuspended in medium. Merozoites were mechanically isolated by passage in a needle.

For P. falciparum merozoites to be subject to conventional glutaraldehyde and osmium fixation or for high pressure freezing and freeze substitution (see below), purification was performed as described by Boyle and colleagues [27].

To obtain $P$. berghei merozoites, blood of an infected mouse was collected in T-medium (RPMI, 20\% FCS, $0.03 \%$ gentamicin $)$ plus heparin, centrifuged $(110 \times g$ for $8 \mathrm{~min})$ and resuspended in T-medium and placed on a shaker $(50 \mathrm{rpm})$ overnight at $37^{\circ} \mathrm{C}$. Mature schizonts were harvested with a Nycodenz gradient (Axis-Shield PoC, Norway), washed and resuspended in RPMI medium prior to freezing. 


\section{Cryo-electron tomography}

Tomography was performed essentially as described before $[15,21,23,24,28]$. Merozoites in RPMI medium mixed with $10 \mathrm{~nm}$ colloidal gold particles were transferred onto glow-discharged holey carbon Quantifoil EM grids. Grids were blotted at $90 \%$ humidity using a Vitrobot (FEI). After removing the liquid excess, the material was rapidly plunge frozen into liquid ethane and stored in liquid nitrogen. The grids were observed in a Titan Krios (FEI) operating at $300 \mathrm{kV}$ and equipped with a Gatan post column energy filter and post-GIF CCDs operating at liquid nitrogen temperature. Tilt series were acquired with an increment of $2^{\circ}$ covering $-60^{\circ}$ to $+60^{\circ}$, with a cumulative dose under 10,000 electrons $/ \mathrm{nm}^{2}$ and a defocus of -3 to $-15 \mu \mathrm{m}$ on a $2 \mathrm{k}$ Ultrascan $1000 \mathrm{CCD}$ camera (Gatan).

For this study a total of 12 (for P. berghei) and 24 (for $P$. falciparum) tomograms were reconstructed by weighted back-projection using the Etomo program in the IMOD software package (Boulder Laboratory for 3D electron microscopy) [29]. Reconstructed tomograms were filtered using non-linear anisotropic diffusion [30]. Visualization, volume rendering and segmentation were performed using the $3 \mathrm{dmod}$ program of the IMOD package [29].

\section{High-pressure freezing and freeze substitution}

Purified P. falciparum merozoites from strain D10 were allowed to invade red blood cells, then $10 \mathrm{~min}$ post invasion were high-pressure frozen using a LeicaEM highpressure freezer. The infected erythrocyte samples were freeze substituted with $1 \%$ uranyl acetate at $-90^{\circ} \mathrm{C}$ for 24 hrs using a Leica AFS automatic freeze substitution machine. Samples were further freeze substituted with acetone and then infiltrated with increasing concentrations of Lowicryl HM20 resin in acetone. Resin was polymerized using UV light treatment for $48 \mathrm{hrs}$ at $-45^{\circ} \mathrm{C}$ then a further $48 \mathrm{hrs}$ at room temperature. Sections of approximately 90 nanometers were cut at room temperature, stained with uranyl acetate and lead citrate and examined using a Philips CM120 BioTWIN transmission electron microscope at $120 \mathrm{kV}$ (Advanced Microscopy facility, School of Botany, the University of Melbourne, Australia).

\section{Conventional transmission electron microscopy}

Purified P. falciparum merozoites (as described above) were fixed in $2.5 \%$ glutaraldehyde in $\mathrm{pH} 7.4$ phosphate buffer on ice. Cells were washed in phosphate buffer and fixed in 1\% osmium tetroxide (ProSciTech, Australia) in $0.1 \mathrm{M}$ phosphate buffer for one hour on ice. Samples were washed in water, then dehydrated in increasing concentrations of ethanol before embedding in LR Gold resin (ProSciTech, Australia) and polymerized using benzoyl peroxide (SPI-Chem, USA). Sections were cut, stained and inspected as described above.

\section{Results and discussion}

In images of merozoites processed in conventional transmission electron microscopy protocols, some organelles are clearly observed such as rhoptries, while even the nucleus can appear in a distorted fashion (Figures 1A-C). This is due to the use of fixative compounds, dehydration and embedding in resin. Better preservation of structural organization can be achieved when specimens are frozen at high pressure and all the steps for transmission electron microscopy are carried out at low temperatures [31]. But even then, where the post-fixation processing is carried out in low temperatures, high-pressure freezing still requires the use of fixative, dehydration and resin, which in turn can also lead to modifications in the structural organization of the merozoites. In addition, the material is stained and uncontrolled, selective depositions of heavy metal salts further obscure fine details just as in classic transmission electron microscopy. Thus, these techniques can lead to misinterpretation. These considerations are not a concern in cryo-electron microscopy, where the parasites are rapidly frozen close to their native state and not subjected to any other procedure that may affect fine structure [20]. However, images of merozoites processed for cryo-electron microscopy, appear more noisy (Figures 1D-F) and it can be more difficult to readily identify the organelles.

Merozoites processed in conventional transmission electron microscopy protocols, ie, room temperature processing, appear to show apicoplasts with three $[16,17]$ or four apparent membranes (Figure 2A). Highpressure frozen merozoites presented four membranes delimiting the apicoplast (Figure 2B). Both in routine (Figure 2A) and in high-pressure (Figure $2 \mathrm{~B}$ ) processing, the apicoplast presented a granular matrix with the first and the second membranes as well as the third and the fourth close to each other. Also, a gap between the second and the third membranes was observed. This general organization is similar to that described previously for the Toxoplasma gondii apicoplast [32].

Reconstructed tomograms of cryo-fixed $P$. berghei merozoites again showed four membranes delimiting the apicoplast (Figures 3 and 4). This is quantitatively observed on the density profiles across an apicoplast, revealing a clear peak for each of the four membranes (Figure 3A) and in a 3D model of the organelle (Figure 3B and Additional file 1: Video 1). It is noteworthy that membranous profiles were also observed within the apicoplast matrix (Figures $3 \mathrm{~A}$ and $3 \mathrm{~B}$ ). The biological nature and purpose of these matrix membranes is unclear, but similar structures have previously been described inside the apicoplast of Plasmodium [16] and Toxoplasma [32]. 

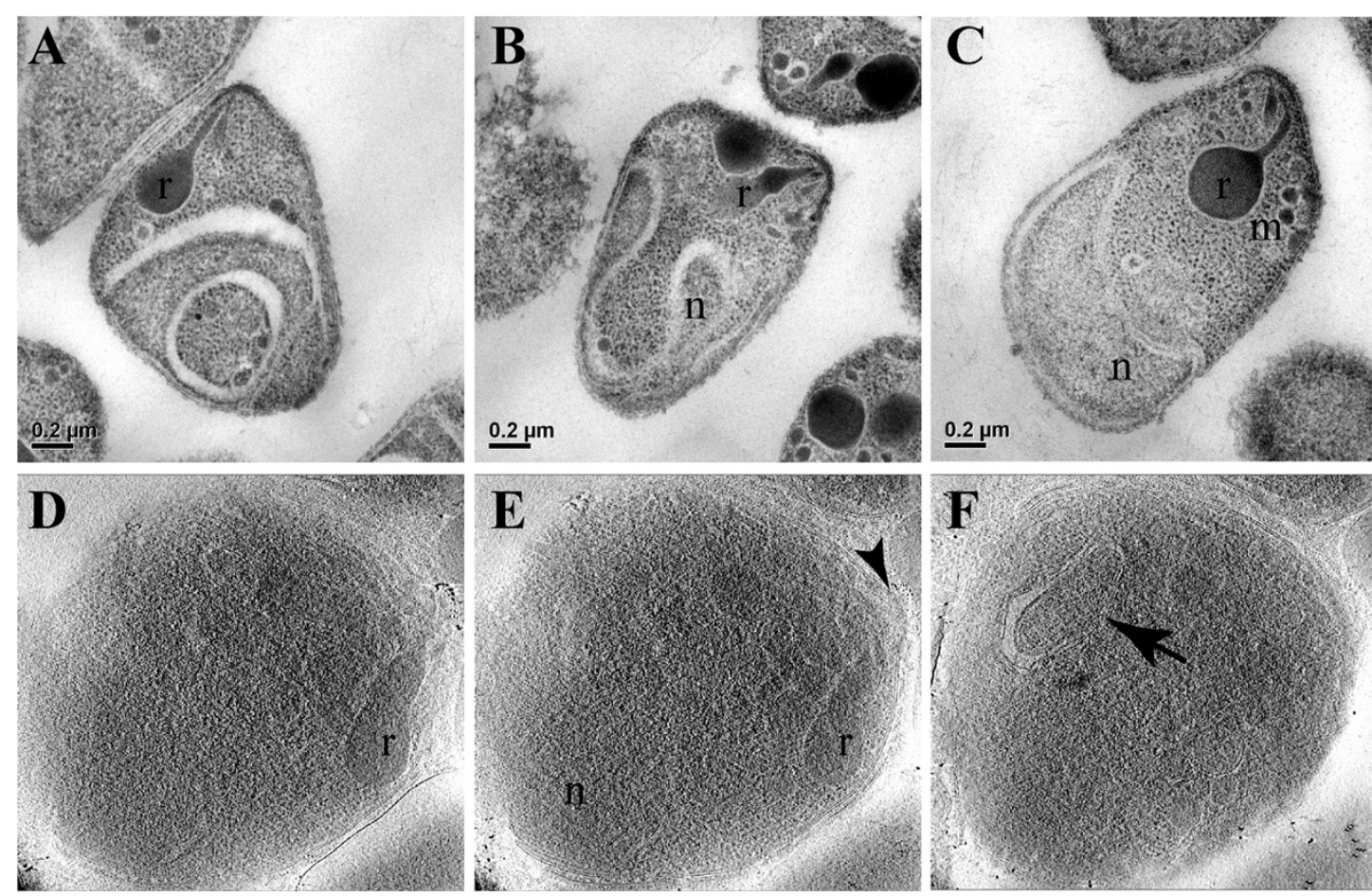

Figure 1 Structural organization of merozoites processed by different electron microscopy techniques. A to $\mathbf{C}$ - different examples of merozoites processed by conventional transmission electron microscopy. Rhoptries $(r)$, micronemes $(m)$ and the nucleus $(n)$ are clearly observed. $\mathbf{D}$ to $\mathbf{F}$ - different sections from a tomogram of a cryo-preserved merozoite. Although more difficult to identify, some structures stand out like the rhoptries ( $r$ ), the polar rings (arrowhead) and the apicoplast (arrow).

Cryo-electron tomography revealed gaps not only between the second and the third membranes (Figures $3 \mathrm{C}$ and $4 \mathrm{~A}$ ) but also, though less frequently, between the third and the fourth membranes (Figure 3D) and the first and the second membranes (Figure 4B).

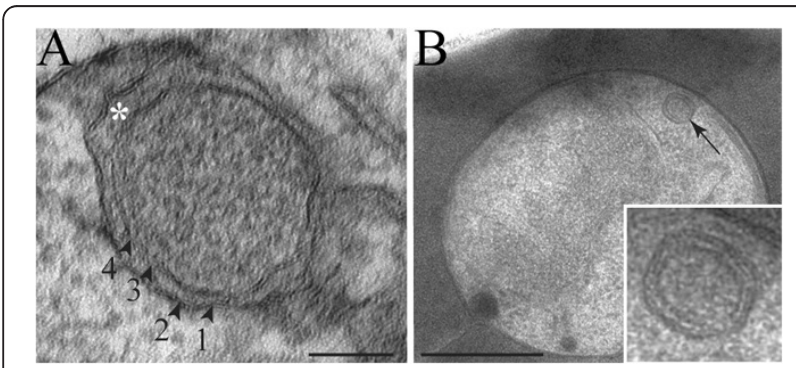

Figure 2 Apicoplast ultrastructure in Plasmodium falciparum merozoites. A - Apicoplast of a Plasmodium falciparum merozoite processed by glutaraldehyde and osmium chemical fixation and examined by transmission electron microscopy. The plastid is clearly surrounded by four membranes (arrowheads), with a gap between the two outermost membranes and the two innermost membranes (asterisk) in some regions. B - When processed by high-pressure freezing and freeze substitution, the apicoplast (arrow) ultrastructure was better preserved, also presenting four membranes. The gap between the membranes 2 and 3 is still present. Scale bars: $A=50$ $\mathrm{nm} ; \mathrm{B}=200 \mathrm{~nm}$.
The apicoplast of $P$. falciparum merozoites (Figure 5) showed the same general morphology as those of $P$. berghei. The apicoplast was not only located close to the nucleus but its outer most membrane was also observed in close apposition to the rhoptries and the mitochondrion (Figures 5B-G). Gaps between the membranes were also observed (Figures 5D and E). Ribosome-like particles were clearly observed in the apicoplast's matrix (Figures $5 \mathrm{C}$ and $5 \mathrm{E}-\mathrm{G})$. Also, large protein complexes were present within the gap between the membranes (Figure 5B). However the high noise in tomograms due to the thickness of the reconstructed volumes (600-800 $\mathrm{nm}$ ) hampered further interpretation of these complexes. Similar gaps and material between membranes was also observed in Eimeria [33].

The relationship between the apicoplast and the endoplasmic reticulum (ER) has been the subject of some conjecture $[16,31,34]$. In heterokonts the plastid resides within the lumen of the ER [35], and a similar arrangement is formally possible for the apicoplast (see reviews in $[14,34,36]$ for more detailed discussions of such models). Such an arrangement might predict that the outer membrane of the plastid contained ribosomes, necessary for the insertion of apicoplast-targeted proteins. As with previous examinations, such ribosomes on the outer membrane of the plastid, and continuities between the membranes of the plastid and ER were not observed. 

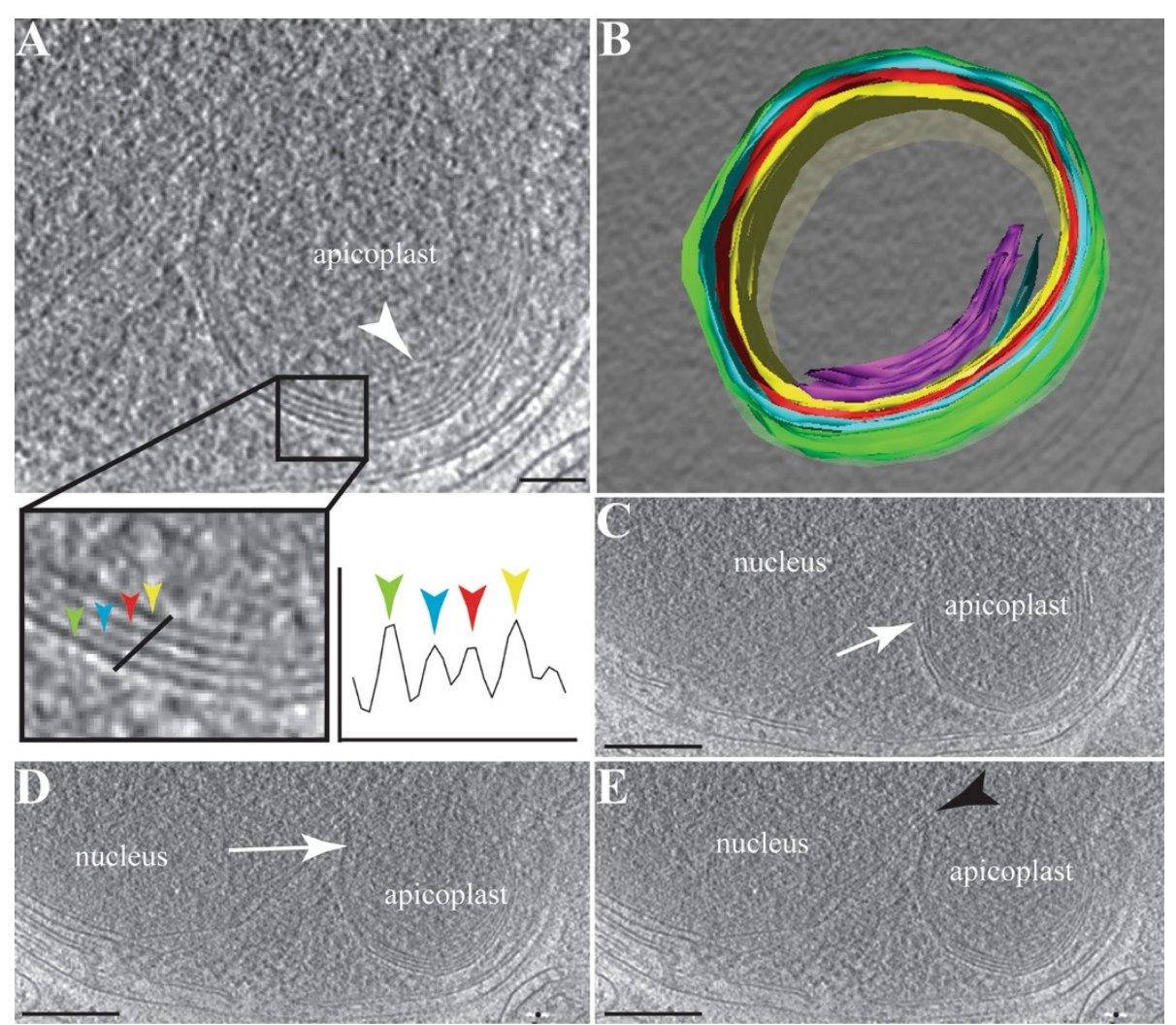

Figure 3 Tomographic reconstruction of the apicoplast in a Plasmodium berghei merozoite. Panel A shows a virtual section of an apicoplast, with the four apicoplast membranes indicated by the coloured arrowheads and a graphed density distribution. The apicoplast matrix presents an internal membranous whorl as indicated (white arrowhead). Panel B shows a rendered model of the apicoplast, highlighting the internal whorls (purple) and the four membranes (yellow, red, blue and green). Panels $\mathbf{C}$ and $\mathbf{D}$ show sections where a gap is observed between membranes 2 and 3 (white arrows). In Panel $\mathbf{E}$, a close apposition is observed between the nucleus and the apicoplast (black arrowhead). Scale bars: $\mathrm{A}=60 \mathrm{~nm} ; \mathrm{B}-\mathrm{E}=20 \mathrm{~nm}$.

However, in merozoites, where all organelles are more closely apposed, the apicoplast was observed in close proximity to the nucleus and ER (Figures $3 \mathrm{E}$ and $4 \mathrm{~A}$ ), as previously described for Plasmodium [16], Toxoplasma [32] and Sarcocystis [11]. Although there appeared to be some contact points with the nuclear envelope and with the ER (Figure 3E), no continuity between these membranes is apparent.

Much has been discussed about how proteins are transported across the apicoplast's surrounding membranes, with various membranous translocon proteins already described [37-41]. In the acquired cryo-

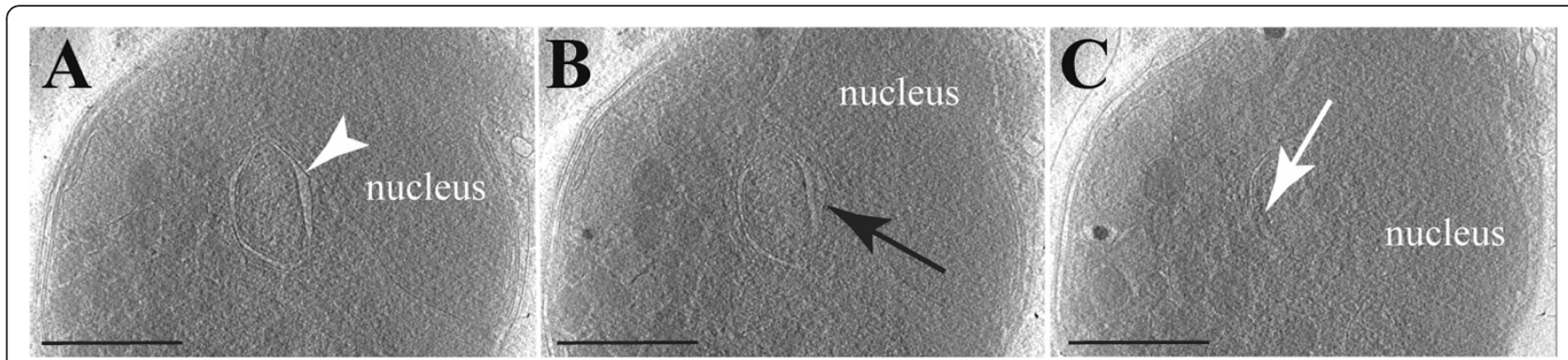

Figure 4 Gaps between apicoplast membranes. A to $\mathbf{C}$ - Different sections of a reconstructed tomogram of a Plasmodium berghei merozoite. The apicoplast with its four membranes is located close to the nucleus. The plastid presents a widening between the two inner membranes and the two outer membranes (white arrowhead). A gap between the two outermost membranes is also occasionally observed (black arrow). In C, electron dense structures (white arrow) are present on some apicoplast membranes, possibly corresponding to protein complexes such as import apparatus. Scale bar $=50 \mathrm{~nm}$. 

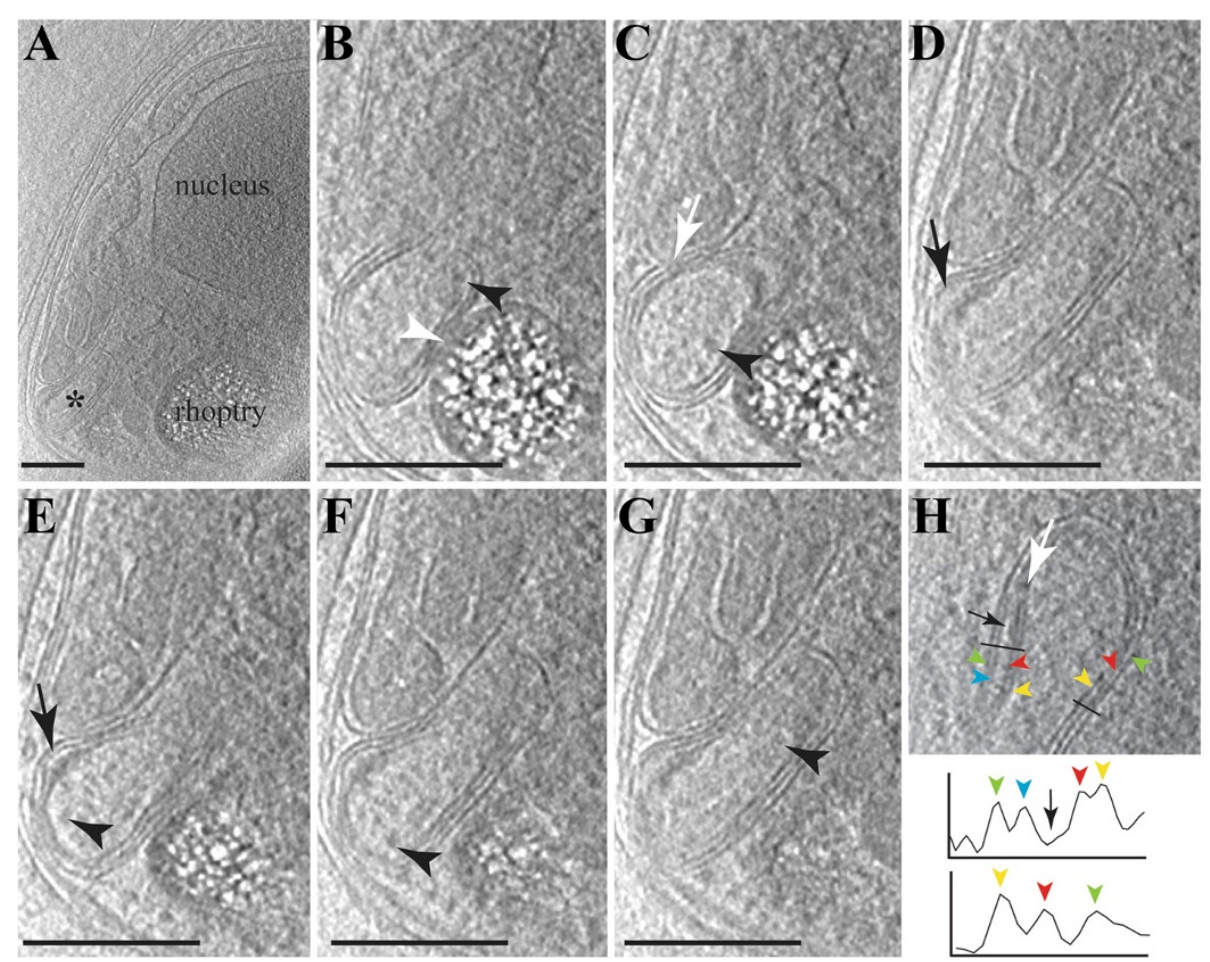

Figure 5 Slices through a reconstructed tomogram of a Plasmodium falciparum merozoite. The apicoplast appears close to the nucleus and the rhoptries. White arrowhead points to an interaction between the apicoplast membrane and the rhoptry membrane. Within the apicoplast matrix, several ribosome-like particles were observed (black arrowheads). Black arrows indicate the different regions with a widening between the two outermost membranes and the two innermost membranes. White arrows indicate electron dense structures (possibly translocons) in the apicoplast membranes. In $\mathbf{H}$, the density distribution over the lines show that the apicoplast has four membranes (top profile), but in some slices through the tomogram only three electron dense lines are apparent (bottom profile), due to the close apposition of two membranes. Scale bars: $\mathrm{A}-\mathrm{G}=25 \mathrm{~nm}$.

tomograms, structures were observed in merozoites' apicoplast of both $P$. berghei and P. falciparum, potentially resembling a translocon (Figures $4 \mathrm{C}$ and $5 \mathrm{C}$ ), although this cannot be resolved with the current images. These features were located between the second and the third membranes.

Going through the virtual sections of a reconstructed tomogram, one can observe that depending on the section the apicoplast can show three or four membranes, or even at the same section both arrangements (Figure 5H). This is usually due to the close apposition of the second membrane with the first one, which can explain the discrepancies in the previously published apicoplast structure descriptions [16-18].

\section{Conclusion}

The Plasmodium apicoplast was shown to contain four membranes as in other apicomplexan parasites. The apicoplast was found in close proximity to the membranes of the nucleus, inner membrane complex, the mitochondrion and rhoptries. The apicoplast membranes are arranged such that the outer and innermost membranes were closely associated to each other while some space occurred between the two doublet membranes. The presented data reconciles molecular evidence as to how transport into the apicoplast is thought to work in Toxoplasma and Plasmodium with electron microscopy data and shows the capacity of cryoelectron tomography to reveal fine structural details in thin parasites.

\section{Additional file}

Additional file 1: Video 1. View of the 3D model from a reconstructed tomogram of a Plasmodium berghei apicoplast. This video is related to Figure 3.

\section{Competing interests}

The authors declare that they have no competing interests.

\section{Authors' contribution}

$L L, M K, S A R, F F$ designed the experiments and carried out data analysis; $L L$, MK, CD, DTR performed the experiments; HS, JB contributed essential materials; LL, SAR, FF wrote the paper. All authors read and approved the final manuscript.

\section{Acknowledgements}

We thank Marek Cyrklaff for discussions, Claudia Kuss for P. falciparum culture, Jessica Kehrer and Mirko Singer for help with $P$. berghei infections and Oliver Grünvogel for helping with the computational modelling. 
The work was funded by the Chica and Heinz Schaller Foundation and an ERC starting grant to FF, a postdoctoral fellowship of the University of Heidelberg Cluster of Excellence CellNetworks to LL, the Swiss initiative for Systems Biology (SystemsX.ch, grant CINA) to MK and HS, a Pratt Foundation postgraduate scholarship through the University of Melbourne to DTR. FF is a member of the European Network of Excellence EVIMalaR. DTR received a travel fellowship from the Australian Society for Biochemistry and Molecular Biology and a Harold Mitchell Travel Fellowship, and CD an OzeMalR travel award. SAR is supported by an ARC Future fellowship (FT0990350).

\section{Author details}

${ }^{1}$ Parasitology, Department of Infectious Diseases, University of Heidelberg Medical School, Im Neuenheimer Feld 324, Heidelberg 69120, Germany. ${ }^{2}$ Center for Cellular Imaging and Nano Analytics (C-CINA), Biozentrum, University of Basel, Mattenstrasse 26, Basel CH-4058, Switzerland. 3 Department of Biochemistry and Molecular Biology, Bio21 Molecular Science and Biotechnology Institute, The University of Melbourne, Victoria 3010, Australia. ${ }^{4}$ Division of Infection and Immunity, The Walter and Eliza Hall Institute of Medical Research, Parkville, VIC 3052, Australia. ${ }^{5}$ Department of Medical Biology, University of Melbourne, Parkville, VIC 3052, Australia.

\section{Received: 22 November 2012 Accepted: 15 January 2013}

Published: 19 January 2013

\section{References}

1. Gould SB, Waller RF, McFadden Gl: Plastid evolution. Annu Rev Plant Biol 2008, 59:491-517.

2. Dodge J: A survey of chloroplast ultrastructure in the Dinophyceae. Phycologia 1975, 14:253-363.

3. Archibald JM: The puzzle of plastid evolution. Curr Biol 2009, 19:R81-R88.

4. Cavalier-Smith T: Principles of protein and lipid targeting in secondary symbiogenesis: euglenoid, dinoflagellate, and sporozoan plastid origins and the eukaryote family tree. J Eukaryot Microbiol 1999, 46:347-366.

5. McFadden Gl, Reith ME, Munholland J, Lang-Unnasch N: Plastid in human parasites. Nature 1996, 381:482

6. Fast NM, Kissinger JC, Roos DS, Keeling PJ: Nuclear-encoded, plastid-targeted genes suggest a single common origin for apicomplexan and dinoflagellate plastids. Mol Biol Evol 2001, 18:418-426.

7. Ralph SA, Van Dooren GG, Waller RF, Crawford MJ, Fraunholz MJ, Foth BJ, Tonkin CJ, Roos DS, McFadden Gl: Metabolic maps and functions of the Plasmodium falciparum apicoplast. Nat Rev Microbiol 2004, 2:203-216.

8. Moore RB, Oborník M, Janouskovec J, Chrudimský T, Vancová M, Green DH, Wright SW, Davies NW, Bolch CJS, Heimann K, Slapeta J, Hoegh-Guldberg O, Logsdon JM, Carter DA: A photosynthetic alveolate closely related to apicomplexan parasites. Nature 2008, 451:959-963.

9. Janouskovec J, Horák A Oborník M, Lukes J, Keeling PJ: A common red algal origin of the apicomplexan, dinoflagellate, and heterokont plastids. Proc Natl Acad Sci USA 2010, 107:10949-10954

10. Kohler S, Delwiche CF, Denny PW, Tilney LG, Webster P, Wilson R, Palme JD, Roos DS: A plastid of probable green algal origin in apicomplexan parasites. Science 1997, 275:1485-1489.

11. Tomova C, Geerts WJC, Müller-Reichert T, Entzeroth R, Humbel BM: New comprehension of the apicoplast of Sarcocystis by transmission electron tomography. Biol Cell 2006, 98:535-545.

12. Diniz JAP, Silva EO, Lainson R, De Souza W: The fine structure of Garnia gonadati and its association with the host cell. Parasitol Res 2000, 86:971-977.

13. Caballero MC, Pedroni MJ, Palmer GH, Suarez CE, Davitt C, Lau AOT: Characterization of acyl carrier protein and LytB in Babesia bovis apicoplast. Mol Biochem Parasitol 2012, 181:125-133.

14. Waller RF, McFadden Gl: The apicoplast: a review of the derived plastid of apicomplexan parasites. Curr Issues Mol Biol 2005, 7:57-79.

15. Kudryashev M, Lepper S, Stanway R, Bohn S, Baumeister W, Cyrklaff M, Frischknecht F: Positioning of large organelles by a membrane associated cytoskeleton in Plasmodium sporozoites. Cell Microbiol 2010, 12:362-371.

16. Hopkins J, Fowler R, Krishna S, Wilson I, Mitchell G, Bannister L: The plastid in Plasmodium falciparum asexual blood stages: a three-dimensional ultrastructural analysis. Protist 1999, 150:283-295.

17. Bannister LH, Hopkins JM, Fowler RE, Krishna S, Mitchell GH: A brief illustrated guide to the ultrastructure of Plasmodium falciparum asexual blood stages. Parasitol Today 2000, 16:427-433.
18. Bannister L, Margos G, Hopkins JM: Making a home for Plasmodium postgenomics: ultrastructural organization of the blood stages. Washington D.C: Molecular Approaches to Malaria. ASM Press; 2005

19. Lepper S, Merkel M, Sartori A, Cyrklaff M, Frischknecht F: Rapid quantification of the effects of blotting for correlation of light and cryo-light microscopy images. J Microsc 2010, 238:21-26.

20. Lučić V, Förster F, Baumeister W: Structural studies by electron tomography: from cells to molecules. Annu Rev Biochem 2005, 74:833-865.

21. Cyrklaff M, Kudryashev M, Leis A, Leonard K, Baumeister W, Ménard R, Meissner M, Frischknecht F: Cryoelectron tomography reveals periodic material at the inner side of subpellicular microtubules in apicomplexan parasites. J Exp Med 2007, 204:1281-1287.

22. Kudryashev M, Lepper S, Baumeister W, Cyrklaff M, Frischknecht F: Geometric constrains for detecting short actin filaments by cryogenic electron tomography. PMC Biophys 2010, 3:6.

23. Kudryashev M, Münter S, Lemgruber L, Montagna G, Stahlberg $H$, Matuschewski K, Meissner M, Cyrklaff M, Frischknecht F: Structural basis for chirality and directional motility of Plasmodium sporozoites. Cell Microbiol 2012, 14:1757-1768.

24. Henrich P, Kilian N, Lanzer M, Cyrklaff M: 3-D analysis of the Plasmodium falciparum Maurer's clefts using different electron tomographic approaches. Biotechnol J 2009, 4:888-894.

25. Cyrklaff M, Sanchez CP, Kilian N, Bisseye C, Simpore J, Frischknecht F, Lanzer M: Hemoglobins S and C Interfere with Actin Remodeling in Plasmodium falciparum-Infected Erythrocytes. Science 2011, 334:1283-1286.

26. Leforestier A, Lemercier N, Livolant F: Contribution of cryoelectron microscopy of vitreous sections to the understanding of biological membrane structure. Proc Natl Acad Sci USA 2012, 109:8959-8964.

27. Boyle MJ, Wilson DW, Richards JS, Riglar DT, Tetteh KKA, Conway DJ, Ralph SA, Baum J, Beeson JG: Isolation of viable Plasmodium falciparum merozoites to define erythrocyte invasion events and advance vaccine and drug development. Proc Natl Acad Sci USA 2010, 107:14378-14383.

28. Kühni-Boghenbor K, Ma M, Lemgruber L, Cyrklaff M, Frischknecht F, Gaschen V, Stoffel M, Baumgartner M: Actin-mediated plasma membrane plasticity of the intracellular parasite Theileria annulata. Cell Microbiol 2012, 14:1867-1879.

29. Kremer JR, Mastronarde DN, McIntosh JR: Computer visualization of threedimensional image data using IMOD. J Struct Bio/ 1996, 116:71-76.

30. Frangakis $A S$, Hegerl R: Noise reduction in electron tomographic reconstructions using nonlinear anisotropic diffusion. J Struct Biol 2001, 135:239-250.

31. Studer D, Humbel BM, Chiquet M: Electron microscopy of high pressure frozen samples: bridging the gap between cellular ultrastructure and atomic resolution. Histochem Cell Biol 2008, 130:877-889.

32. Tomova C, Humbel BM, Geerts WJC, Entzeroth R, Holthuis JCM, Verkleij AJ: Membrane contact sites between apicoplast and ER in Toxoplasma gondii revealed by electron tomography. Traffic 2009, 10:1471-1480.

33. Ferguson DJ, Campbell SA, Henriquez FL, Phan L, Mui E, Richards TA, Muench SP, Allary M, Lu JZ, Prigge ST, Tomley F, Shirley MW, Rice DW, McLeod R, Roberts CW: Enzymes of type II fatty acid synthesis and apicoplast differentiation and division in Eimeria tenella. Int J Parasitol 2007, 37:33-51.

34. Tonkin CJ, Kalanon M, McFadden Gl: Protein targeting to the malaria parasite plastid. Traffic 2008, 9:166-175

35. Gibbs SP: The route of entry of cytoplasmically synthesized proteins into chloroplasts of algae possessing chloroplast ER. J Cell Sci 1979, 35:253-266

36. Roos DS, Crawford MJ, Donald RG, Kissinger JC, Klimczak LJ, Striepen B: Origin, targeting, and function of the apicomplexan plastid. Curr Opin Microbiol 1999, 2:426-432

37. Kalanon M, Tonkin CJ, McFadden Gl: Characterization of two putative protein translocation components in the apicoplast of Plasmodium falciparum. Eukaryot Cell 2009, 8:1146-1154.

38. Van Dooren GG, Tomova C, Agrawal S, Humbel BM, Striepen B: Toxoplasma gondii Tic20 is essential for apicoplast protein import. Proc Natl Acad SCi USA 2008, 105:13574-13579.

39. Brooks CF, Johnsen H, Van Dooren GG, Muthalagi M, Lin SS, Bohne W, Fischer K, Striepen B: The Toxoplasma apicoplast phosphate translocator links cytosolic and apicoplast metabolism and is essential for parasite survival. Cell Host Microbe 2010, 7:62-73. 
40. Karnataki A, DeRocher A, Coppens I, Nash C, Feagin JE, Parsons M: Cell cycle-regulated vesicular trafficking of Toxoplasma APT1, a protein localized to multiple apicoplast membranes. Mol Microbiol 2007 63:1653-1668.

41. Glaser S, Higgins MK: Overproduction, purification and crystallization of PfTic22, a component of the import apparatus from the apicoplast of Plasmodium falciparum. Acta Crystallogr Sect F Struct Biol Cryst Commun 2012, 68:351-354.

doi:10.1186/1475-2875-12-25

Cite this article as: Lemgruber et al:: Cryo-electron tomography reveals four-membrane architecture of the Plasmodium apicoplast. Malaria Journal 2013 12:25.

\section{Submit your next manuscript to BioMed Central and take full advantage of:}

- Convenient online submission

- Thorough peer review

- No space constraints or color figure charges

- Immediate publication on acceptance

- Inclusion in PubMed, CAS, Scopus and Google Scholar

- Research which is freely available for redistribution 